\title{
Health-related quality of life of carfilzomib- and daratumumab-based therapies in patients with relapsed/refractory multiple myeloma, based on German benefit assessment data
}

\author{
Katja Weisel ${ }^{1,2} \cdot$ Heinz Ludwig $^{3} \cdot$ Achim Rieth $^{4} \cdot$ Andrea Lebioda $^{4} \cdot$ Hartmut Goldschmidt $^{5}$
}

Accepted: 14 September 2019 / Published online: 24 September 2019

(c) The Author(s) 2019

\begin{abstract}
Background Carfilzomib and daratumumab are licensed in relapsed/refractory multiple myeloma (RRMM), but no headto-head trials have been conducted.

Methods We used data from dossiers prepared for the German Federal Joint Committee based on two phase III randomized trials of carfilzomib-based therapies (ASPIRE, ENDEAVOR) and two of daratumumab-based therapies (POLLUX, CASTOR) to conduct a descriptive assessment of health-related quality of life (HRQoL). HRQoL was assessed using the European Organisation for Research and Treatment of Cancer 30-item HRQoL Questionnaire, with hazard ratios calculated for carfilzomib- and daratumumab-based therapy versus comparators for time to $\mathrm{HRQoL}$ deterioration of $\geq 10$ points. Analyses were also conducted on data from the EORTC 20-item myeloma-specific questionnaire, the Functional Assessment of Cancer Therapy/Gynecologic Oncology Group-Neurotoxicity scale, and the visual analog scale of the EuroQoL 5-dimension, 5-level questionnaire, where results for these instruments were available. As the designs and patient population of the four trials were similar but not identical, the analysis included only indirect, descriptive comparisons.

Results Compared with lenalidomide/dexamethasone, median time to deterioration in global health status/QoL was longer for carfilzomib-based therapy versus control, but similar for daratumumab-based therapy and control. Compared with bortezomib/dexamethasone, time to deterioration was significantly longer for carfilzomib-based therapy versus control for global health status/QoL and numerous functional and symptom subscales. HRQoL measurement is feasible in large RRMM populations.
\end{abstract}

Conclusion Descriptive assessment of HRQoL data suggests potential benefits for carfilzomib-based over daratumumabbased therapy.

Keywords Quality of life · Daratumumab · Carfilzomib $\cdot$ Multiple myeloma $\cdot$ Indirect comparison

Electronic supplementary material The online version of this article (https://doi.org/10.1007/s11136-019-02307-5) contains supplementary material, which is available to authorized users.

Katja Weisel

k.weisel@uke.de

1 Medizinische Klinik und Poliklinik (Onkologie, Hämatologie, Knochenmarktransplantation mit Abteilung für Pneumologie), Universitätsklinikum Hamburg Eppendorf, II., Martinistr. 52, 20246 Hamburg, Germany

2 Department of Medicine II, University Hospital Tubingen, Tübingen, Germany

\section{Introduction}

Multiple myeloma (MM) is an incurable, relapsing disease that is generally diagnosed in elderly individuals; median age at diagnosis is 72 years for men and 74 years for women [1-3]. In recent years, mortality rates in MM have improved

3 1st Department of Medicine, Wilhelminen Cancer Research Institute, Vienna, Austria

4 Amgen $\mathrm{GmbH}$, Munich, Germany

5 Internal Medicine $\mathrm{V}$ and National Center for Tumor Diseases (NCT), University Clinic Heidelberg, Heidelberg, Germany 
significantly as a result of advances in treatment [4]. The aim of treatment has therefore shifted from purely palliative therapy to the early use of potent treatments to prolong disease control and improve overall survival (OS) [5-8].

The World Health Organization defines quality of life $(\mathrm{QoL})$ as "an individual's perception of their position in life in the context of the culture and value systems in which they live and in relation to their goals, expectations, standards and concerns" [9]. Health-related QoL (HRQoL) can be additionally defined as the functional effect of a medical condition and its consequent therapy upon a patient [10]. Patients with MM often experience substantial adverse effects on HRQoL, including pain, fatigue, and breathlessness, as well as impaired physical functioning [11-13]. Furthermore, treatment-related toxicity can also negatively affect patients' HRQoL [14]. During treatment, HRQoL is generally maintained at baseline levels or declines, although improvements in some HRQoL domains may be seen [15-19]. As a result, QoL has become an important determinant of therapy, with some physicians and patients choosing to optimize QoL at the cost of prolonged survival [20]. QoL is also a central component of health technology and cost-effectiveness assessment [21]. Incorporation of QoL endpoints in clinical trials is therefore essential to allow better clinical decisionmaking in patients with MM [22], including those with relapsed/refractory (RR) MM.

Carfilzomib is an epoxyketone proteasome inhibitor that binds selectively and irreversibly to the constitutive proteasome and immunoproteasome. In the phase III ASPIRE and ENDEAVOR trials, carfilzomib-based therapy was associated with significantly prolonged progression-free survival (PFS) and OS compared with controls [5, 8, 23, 24]. Based on these two studies, carfilzomib is licensed in Europe and the USA in combination with either lenalidomide and dexamethasone or dexamethasone alone for the treatment of adults with RRMM who have received at least one prior therapy $[25,26]$. Furthermore, the European Summary of Product Characteristics notes the benefits of carfilzomib on HRQoL as reported in the ASPIRE and ENDEAVOR studies. In the USA, carfilzomib is also licensed as monotherapy in patients with RRMM who have received one or more lines of therapy [26].

Daratumumab is a monoclonal antibody specific for CD38, which is overexpressed in hematological malignancies such as MM [27]. The phase III CASTOR and POLLUX trials of combinations including daratumumab showed significantly prolonged PFS versus controls [28, 29]. As a result, daratumumab is licensed in Europe as monotherapy for the treatment of adults with RRMM whose prior therapy included a proteasome inhibitor and an immunomodulatory agent and who have progressed while receiving their most recent therapy. It is also licensed in combination with lenalidomide/dexamethasone or bortezomib/dexamethasone for the treatment of adults with MM who have received at least one prior therapy [30]. In the USA, daratumumab is licensed: (1) in combination with bortezomib, melphalan and prednisone for the treatment of patients with newly diagnosed MM who are ineligible for autologous stem cell transplant; (2) in combination with lenalidomide and dexamethasone, or bortezomib and dexamethasone, for the treatment of patients with MM who have received at least one prior therapy; (3) in combination with pomalidomide and dexamethasone for the treatment of patients with MM who have received at least two prior therapies including lenalidomide and a proteasome inhibitor; and (4) as monotherapy for the treatment of patients with MM who have received at least three prior lines of therapy including a proteasome inhibitor and an immunomodulatory agent or who are doublerefractory to both of these drug classes [31].

To date, there have been no direct comparisons between carfilzomib and daratumumab to permit an assessment of their relative impacts on HRQoL, or efficacy and tolerability endpoints. However, both carfilzomib and daratumumab have recently gone through the German Federal Joint Committee (Gemeinsamer Bundesausschuss; G-BA) early benefit assessment process according to $\$ 35$ a social code book $\mathrm{V}$ (under Arzneimittelmarkt-Neuordnungsgesetz [AMNOG; 'Pharmaceuticals Market Reorganisation Act']) and the respective dossiers prepared by the pharmaceutical companies are publicly available. Here, we used these publicdomain dossier data to conduct a descriptive, indirect comparison of carfilzomib and daratumumab and their effects on HRQoL as reported from the ASPIRE, ENDEAVOR, CASTOR, and POLLUX trials [32, 33].

\section{Methods}

\section{Trial designs}

All four trials were phase III, randomized, open-label trials including patients with RRMM [5, 8, 23, 24, 28, 29, 32-37] (Table 1). All four trials were conducted in accordance with the Declaration of Helsinki and all participants provided written, informed consent. The primary endpoint in each trial was PFS, with OS and HRQoL assessed prospectively as secondary endpoints.

The efficacy and safety of carfilzomib-based therapy were evaluated in the ASPIRE and ENDEAVOR studies. In ASPIRE (ClinicalTrials.gov: NCT01080391; EudraCT: 2009-016839-35), patients received lenalidomide (25 mg) and dexamethasone $(40 \mathrm{mg}$ ), with or without carfilzomib $\left(20 \mathrm{mg} / \mathrm{m}^{2}\right.$ on days 1 and 2 of cycle $1 ; 27 \mathrm{mg} / \mathrm{m}^{2}$ thereafter twice weekly with the frequency reduced to once every 2 weeks after 12 cycles) [24]. In ENDEAVOR (ClinicalTrials.gov: NCT01568866; EudraCT: 2012-000128-16), 


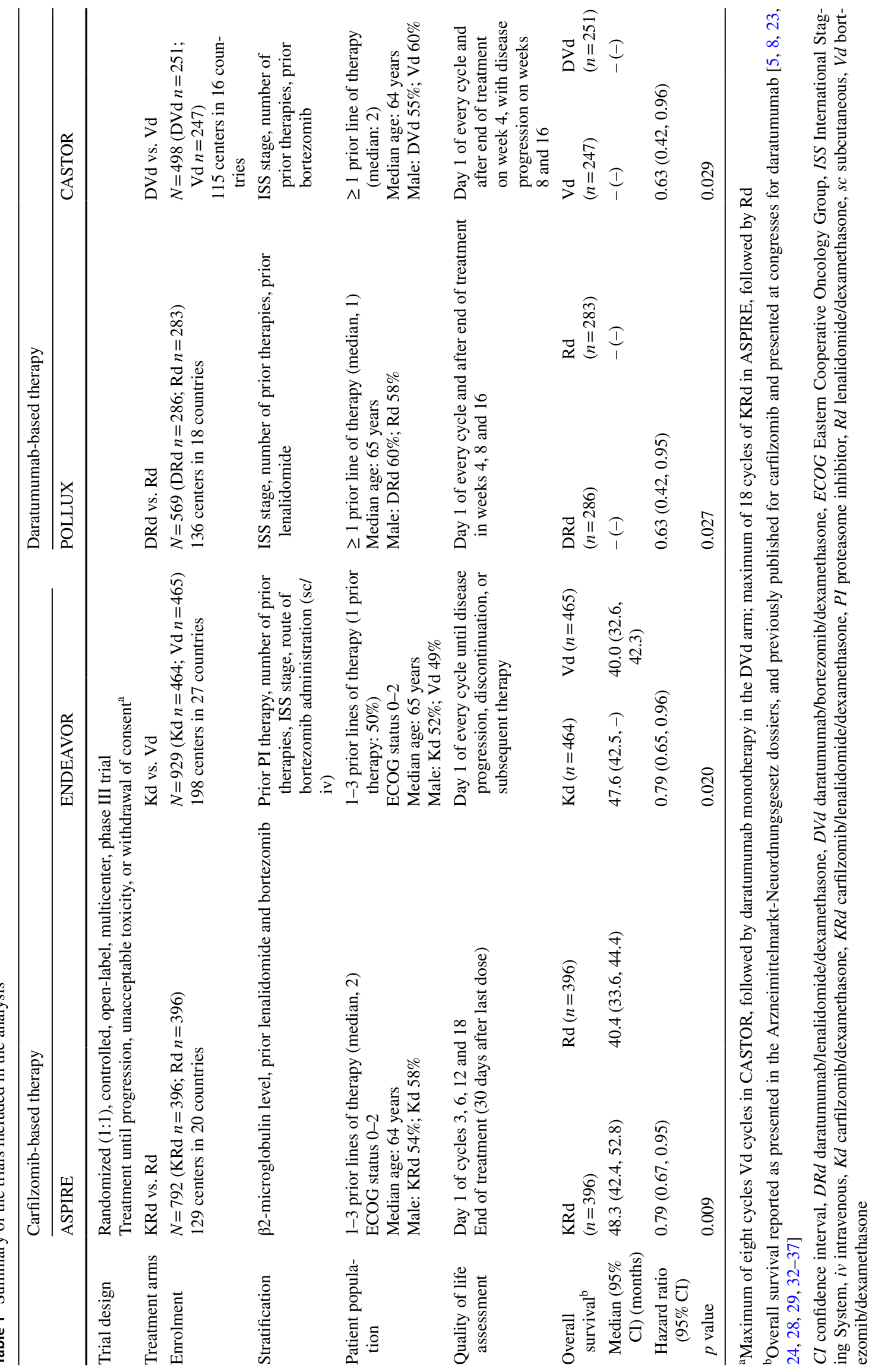


patients received dexamethasone $(20 \mathrm{mg})$ with either carfilzomib $\left(20 \mathrm{mg} / \mathrm{m}^{2}\right.$ on days 1 and 2 of cycle $1 ; 56 \mathrm{mg} / \mathrm{m}^{2}$ thereafter) or bortezomib $\left(1.3 \mathrm{mg} / \mathrm{m}^{2}\right)$ [23].

Daratumumab-based treatment was evaluated in the POLLUX and CASTOR studies. In POLLUX (ClinicalTrials. gov: NCT02076009; EudraCT: 2013-005525-23), patients received lenalidomide $(25 \mathrm{mg})$ and dexamethasone $(40 \mathrm{mg})$, with or without daratumumab $(16 \mathrm{mg} / \mathrm{kg}$ given weekly for 8 weeks, followed by dosing every 2 weeks for 16 weeks, and every 4 weeks thereafter) [28], while in CASTOR (ClinicalTrials.gov: NCT02136134; EudraCT: 2014-000255-85), patients received dexamethasone $(20 \mathrm{mg})$ and bortezomib $\left(1.3 \mathrm{mg} / \mathrm{m}^{2}\right)$, with or without daratumumab $(16 \mathrm{mg} / \mathrm{kg}$ given weekly for 9 weeks, every 3 weeks for 15 weeks, and every 4 weeks thereafter) [29].

Treatment generally continued until disease progression, unacceptable toxicity, or withdrawal of consent. However, in CASTOR, a maximum of eight cycles of bortezomib and dexamethasone was permitted. Similarly, in ASPIRE, only 18 cycles of carfilzomib, lenalidomide, and dexamethasone were permitted, followed by lenalidomide plus dexamethasone $[23,24,28,29]$.

\section{Quality of life assessment}

The European Organisation for Research and Treatment of Cancer (EORTC) 30-item QoL Questionnaire (QLQ-C30) was used in all four trials to assess HRQoL [32, 33, 35, 36]. This questionnaire, which includes both specific functional and symptom subscales as well as an assessment of global health status, has been extensively validated and is widely used for assessment of HRQoL in patients with cancer [38]. In ASPIRE and ENDEAVOR, HRQoL was also assessed using the EORTC 20-item myeloma-specific questionnaire (QLQ-MY20) and, in ENDEAVOR only, the Functional Assessment of Cancer Therapy/Gynecologic Oncology Group-Neurotoxicity scale (FACT/GOG-Ntx) was also used. In addition, HRQoL was assessed in CASTOR and POLLUX using the visual analog scale of the EuroQoL 5-dimension, 5-level questionnaire (EQ-5D-VAS). HRQoL was assessed on day 1 of some or all cycles, as well as at other pre-planned timepoints, depending on the trial (Table 1).

\section{Data synthesis and analysis}

All reported data are derived from public-domain dossiers, as part of the AMNOG assessment by G-BA. Adherence to HRQoL assessment was recorded throughout the studies and return rates calculated for each questionnaire, based on the number of patients alive and receiving study treatment for each trial. Hazard ratios (HRs) were calculated for carfilzomib- and daratumumab-based therapy versus comparators for time to HRQoL deterioration of $\geq 10$ points on the EORTC QLQ-C30. For carfilzomib, 95\% confidence intervals (CIs) were also calculated. In ASPIRE and ENDEAVOR, time to a $\geq 10$-point deterioration was also assessed on the EORTC QLQ-MY20. Time to a $\geq 5$-point deterioration on the FACT/GOG-Ntx was evaluated for ENDEAVOR, and time to a $\geq 7$-point deterioration on the EQ-5D-VAS assessed in POLLUX and CASTOR. Calculations were based on intention-to-treat (ITT) populations. The minimal important difference (MID) for the EORTC QLQ$\mathrm{C} 30$ has been reported as $8-12$ points in patients with $\mathrm{MM}$ [39], and an MID of 10 points is recommended for identifying clinically relevant differences in worsening of HRQoL [40]. Furthermore, patients with an increase of $\geq 10$ points generally report a subjective improvement of 'moderate' or better [39, 41, 42].

For completeness, summary data for PFS and OS from these studies are included, as have been reported previously [5, 8, 34, 37]. In brief, PFS and OS were analyzed by the Kaplan-Meier method, and median PFS and OS were calculated with $95 \%$ CIs for study treatment and comparator. HRs and $95 \%$ CIs were also calculated for time to first adverse event, serious adverse event, severe adverse event (Grade $\geq 3$ ), discontinuation from any study medication, and discontinuation from all study medications. For the ENDEAVOR head-to-head comparison, relative risks and 95\% CIs for time to occurrence of peripheral neuropathy symptoms Grade $\geq 2$ and Grade $\geq 3$ were also calculated.

As the designs and patient population of the four trials were similar but not identical (Table 1), the present analysis includes only indirect, descriptive comparisons between carfilzomib- and daratumumab-based therapy.

\section{Results}

\section{Patient population and return rates for quality of life questionnaires}

In total, 2788 patients across the four studies were included in the analyses. Patient numbers in the ITT population for each treatment arm in the four studies are shown in Table 1. Return rates for HRQoL questionnaires were $>84 \%$ at baseline and remained high throughout treatment in all four trials (Supplemental Table 1).

\section{Quality of life: carfilzomib-based therapy versus lenalidomide/dexamethasone}

Median time to $\geq 10$-point deterioration on the EORTC QLQ-C30 subscales is shown in Table 2. In ASPIRE, median time (in months) to deterioration in global health status/QoL was consistently longer for carfilzomib-based therapy versus control. Results on the global health 
Table 2 Time to $\geq 10$-point deterioration on the EORTC QLQ-C30 and subscales with carfilzomib-related therapy (ASPIRE and ENDEAVOR) and daratumumab-based therapy (POLLUX and CASTOR) versus comparator

\begin{tabular}{|c|c|c|c|c|c|c|c|c|}
\hline & \multicolumn{2}{|l|}{ ASPIRE $^{\mathrm{a}}$} & \multicolumn{2}{|l|}{ ENDEAVOR $^{\mathrm{a}}$} & \multicolumn{2}{|l|}{ POLLUX $^{\mathrm{b}}$} & \multicolumn{2}{|c|}{ CASTOR $^{b}$} \\
\hline & $\begin{array}{l}\text { KRd } \\
(n=396)\end{array}$ & $\begin{array}{l}\mathrm{Rd} \\
(n=396)\end{array}$ & $\begin{array}{l}\mathrm{Kd} \\
(n=464)\end{array}$ & $\begin{array}{l}\mathrm{Vd} \\
(n=465)\end{array}$ & $\begin{array}{l}\text { DRd } \\
(n=286)\end{array}$ & $\begin{array}{l}\mathrm{Rd} \\
(n=283)\end{array}$ & $\begin{array}{l}\text { DVd } \\
(n=251)\end{array}$ & $\begin{array}{l}\mathrm{Vd} \\
(n=247)\end{array}$ \\
\hline \multicolumn{9}{|l|}{ Functional scales } \\
\hline Global health status/QoL & $16.6(15.9,-)$ & $11.9(10.3,-)$ & $3.8(2.9,4.7)$ & $2.8(2.8,3.5)$ & 4.7 & 4.7 & 3.5 & 3.7 \\
\hline Emotional functioning & $18.5(16.4,-)$ & $-(16.2,-)$ & $7.0(5.6,11.2)$ & $6.4(4.7,7.5)$ & 6.6 & 7.8 & 5.7 & 4.4 \\
\hline Social functioning & $15.9(10.3,16.6)$ & $10.3(4.9,15.9)$ & $2.8(2.8,3.8)$ & $2.8(2.8,3.7)$ & 3.8 & 2.9 & 3.0 & 3.0 \\
\hline Cognitive functioning & $11.3(10.3,15.9)$ & $10.5(6.1,15.9)$ & $4.7(3.8,6.6)$ & $3.8(2.9,4.9)$ & 4.9 & 4.6 & 3.5 & 3.4 \\
\hline Physical functioning & $10.3(5.2,15.9)$ & $10.3(5.7,15.8)$ & $5.6(4.7,7.5)$ & $3.8(3.3,5.6)$ & 5.9 & 7.5 & 4.3 & 4.2 \\
\hline Role functioning & $17.1(16.4,21.3)$ & $15.9(10.5,16.4)$ & $2.8(1.9,2.9)$ & $2.8(2.2,3.3)$ & 3.7 & 3.1 & 2.3 & 2.8 \\
\hline \multicolumn{9}{|l|}{ Symptom scales } \\
\hline Fatigue & $4.7(4.7,10.3)$ & $5.7(4.7,10.5)$ & $1.9(1.9,2.0)$ & $1.9(1.8,2.6)$ & 1.9 & 2.0 & 1.6 & 2.1 \\
\hline Pain & $16.1(10.9,17.0)$ & $16.0(11.0,-)$ & $5.6(4.7,7.1)$ & $4.0(3.5,5.6)$ & 5.6 & 5.6 & 3.5 & 3.7 \\
\hline Nausea/vomiting & $21.3(16.5,21.3)$ & $17.2(17.2,-)$ & $17.9(11.2,-)$ & $8.4(6.6,12.0)$ & 13.9 & 10.3 & 7.3 & - \\
\hline Dyspnea & $16.4(15.9,-)$ & $17.3(15.0,-)$ & $2.9(2.8,3.8)$ & $3.8(2.9,4.9)$ & 5.5 & 5.7 & 3.5 & 2.9 \\
\hline Insomnia & $15.9(10.3,16.3)$ & $15.9(10.3,16.2)$ & $3.7(2.8,4.7)$ & $2.8(1.9,3.5)$ & 6.6 & 3.7 & 2.4 & 2.9 \\
\hline Appetite loss & $16.5(16.1,-)$ & $-(16.4,-)$ & $11.2(9.4,-)$ & $5.5(4.6,6.9)$ & 7.2 & 10.2 & 5.0 & 5.9 \\
\hline Diarrhea & $15.9(10.5,15.9)$ & $15.9(10.8,16.3)$ & $10.3(8.4,15.1)$ & $5.6(4.7,7.5)$ & 5.6 & 5.7 & 5.7 & 6.9 \\
\hline Constipation & $17.5(16.6,-)$ & $16.1(10.6,-)$ & $-(15.2,-)$ & $4.7(3.6,7.3)$ & 4.7 & 3.3 & - & 7.3 \\
\hline
\end{tabular}

${ }^{a}$ Data are presented as median ( $95 \%$ confidence interval) months (calculated as days/30)

${ }^{b}$ Data are presented as median months. No $95 \%$ confidence intervals were presented for daratumumab data in the dossiers

Statistically significant results (see Figs. 1, 2, 3 and 4) are highlighted in bold

DRd daratumumab/lenalidomide/dexamethasone, $D V d$ daratumumab/bortezomib/dexamethasone, EORTC European Organisation for Research and Treatment of Cancer, $K d$ carfilzomib/dexamethasone, $K R d$ carfilzomib/lenalidomide/dexamethasone, $Q o L$ quality of life, $Q L Q$-C30 EORTC 30-item Quality of Life Questionnaire, $R d$ lenalidomide/dexamethasone, $V d$ bortezomib/dexamethasone

status/QoL, physical functioning, and constipation subscales significantly favored carfilzomib-based therapy, while appetite loss favored lenalidomide/dexamethasone (Fig. 1; Table 2). There were no significant differences between treatment arms in any of the other subscales of the EORTC QLQ-C30, nor on the EORTC QLQ-MY20 (Fig. 1).

\section{Quality of life: daratumumab-based therapy versus lenalidomide/dexamethasone}

Median time to $\geq 10$-point deterioration on the EORTC QLQ-C30 subscales is shown in Table 2. In POLLUX, median time to deterioration in global health status/QoL did not differ between daratumumab-based therapy and control. Results on the social functioning subscale significantly favored daratumumab-based therapy, with no significant differences between treatment arms on any other subscale (Fig. 2; Table 2). In addition, there were no significant differences between treatment arms on the EQ-5D-VAS (HR 0.97; 95\% CI 0.78, 1.21).

\section{Quality of life: carfilzomib-based therapy versus bortezomib/dexamethasone}

Median times to $\geq 10$-point deterioration in the EORTC QLQ-C30 subscales are shown in Table 2. In ENDEAVOR there were significant differences favoring carfilzomibbased therapy in global health status/QoL and on numerous functional (social, cognitive and physical) and symptom (insomnia, appetite loss, nausea and vomiting, diarrhea, and constipation) subscales of the EORTC QLQC30 (Fig. 3; Table 2). There were no significant differences between treatment arms in the remaining subscales. In this trial, treatment continued until disease progression (a median of eight cycles bortezomib/dexamethasone was given) (Table 1). On the EORTC QLQ-MY20, there was a significant difference in favor of carfilzomib-based therapy on the side effects subscale, but not the disease symptoms, future perspective, or body image subscales (Supplemental Fig. 1), and on the FACT/GOG-NTx, there was a significant difference in favor of carfilzomib-based therapy versus the comparator arm (HR $0.84 ; 95 \%$ CI $0.40,1.28$; $p<0.001$ ). 
Fig. 1 Forest plot showing hazard ratios for differences between lenalidomide/ dexamethasone and carfilzomib/ lenalidomide/dexamethasone (ASPIRE trial) for time to $\geq 10$-point deterioration on the EORTC QLQ-C30 and in subscales of the EORTC QLQMY20. CI confidence interval, EORTC European Organisation for Research and Treatment of Cancer, $Q L Q-C 30$ EORTC 30-item Quality of Life Questionnaire, $Q L Q-M Y 20$ EORTC 20-item myeloma-specific questionnaire, $Q o L$ quality of life
$\frac{\mathrm{N}}{\text { (active/comparator) }}$

Hazard ratio $\quad \mathrm{P}$-value

\section{EORTC QLQ-C30}

Global health status/QoL

Emotional functioning

Social functioning

Cognitive functioning

Physical functioning

Role functioning

Fatigue

Pain

Nausea and vomiting

Dyspnea

Insomnia

Appetite loss

Diarrhea

Constipation

EORTC QLQ-MY20

Disease symptoms

Side effects of treatment

Future perspective

Body image

$144 / 152$
$124 / 118$
$171 / 174$
$184 / 162$
$141 / 146$
$186 / 171$
$211 / 188$
$159 / 140$
$108 / 94$
$151 / 131$
$167 / 150$
$135 / 94$
$181 / 136$
$113 / 139$

$130 / 122$

$157 / 141$

$266 / 216$

$104 / 100$
$(95 \% \mathrm{CI})$

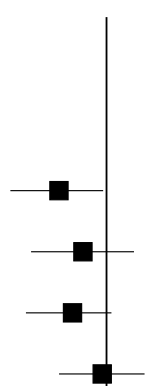

$0.90(0.70,1.16) \quad 0.436$

$0.85(0.68,1.04) \quad 0.119$

$0.99(0.80,1.22) \quad 0.904$

$0.79(0.63,1.00) \quad 0.503$

$0.96(0.78,1.19) \quad 0.730$

$1.05(0.86,1.28) \quad 0.624$

$0.97(0.77,1.22) \quad 0.791$

$0.93(0.71,1.23) \quad 0.630$

$1.02(0.80,1.29) \quad 0.882$

$0.92(0.74,1.15) \quad 0.460$

$1.32(1.01,1.71) \quad 0.043$

$1.11(0.89,1.39) \quad 0.350$

$0.68(0.53,0.87) \quad 0.003$

$0.86(0.67,1.11) \quad 0.244$

$1.00(0.79,1.25) \quad 0.975$

$1.17(0.98,1.40) \quad 0.081$

$0.90(0.69,1.19) \quad 0.975$

Favors carfilzomib-based therapy

\section{Quality of life: daratumumab-based therapy versus bortezomib/dexamethasone}

Median times to $\geq 10$-point deterioration in the EORTC QLQ-C30 subscales are shown in Table 2. In CASTOR there were no significant differences on any subscales of the EORTC QLQ-C30 between daratumumab-based therapy and control (Fig. 4; Table 2), and no significant difference on the EQ-5D-VAS (HR 1.00; 95\% CI 0.79, 1.28).

\section{Overall and progression-free survival and adverse events}

All four trials reported significantly improved HRs for OS for study treatment (carfilzomib- or daratumumab-based therapy) versus control (Table 1). In ASPIRE, median OS was 48.3 months with carfilzomib/lenalidomide/dexamethasone versus 40.4 months with lenalidomide/dexamethasone [8], and in ENDEAVOR, median OS was 47.6 months with carfilzomib/dexamethasone versus 40.0 months with bortezomib/dexamethasone [5] (Table 1). In POLLUX and CASTOR, however, data were immature and, as a result, median OS was not reached in any treatment arm (Table 1).

Significant PFS benefits for study treatment versus control were also reported in all four trials. Median PFS in ASPIRE was 26.3 months with carfilzomib/lenalidomide/ dexamethasone versus 17.6 months with lenalidomide/dexamethasone (HR 0.69; 95\% CI 0.57, 0.83; $p<0.0001$ ) [24, 33 ], while in ENDEAVOR median PFS was 18.7 months with carfilzomib/dexamethasone versus 9.4 months with bortezomib/dexamethasone (HR 0.53; 95\% CI 0.44, 0.65; $p<0.0001$ ) [23]. Median PFS was not reached with daratumumab/lenalidomide/dexamethasone in POLLUX and with daratumumab/bortezomib/dexamethasone in CASTOR [34, 37]. Median PFS with lenalidomide/dexamethasone (POLLUX) and daratumumab/bortezomib/dexamethasone (CASTOR) was 17.5 months (HR 0.37; 95\% CI 0.28, 0.50; 
Fig. 2 Forest plot showing hazard ratios for differences between lenalidomide/dexamethasone and daratumumab/ lenalidomide/dexamethasone (POLLUX trial) for time to $\geq 10$-point deterioration on the EORTC QLQ-C30. CI confidence interval, EORTC European Organisation for Research and Treatment of Cancer, $Q L Q-C 30$ EORTC 30-item Quality of Life Questionnaire, $Q o L$ quality of life

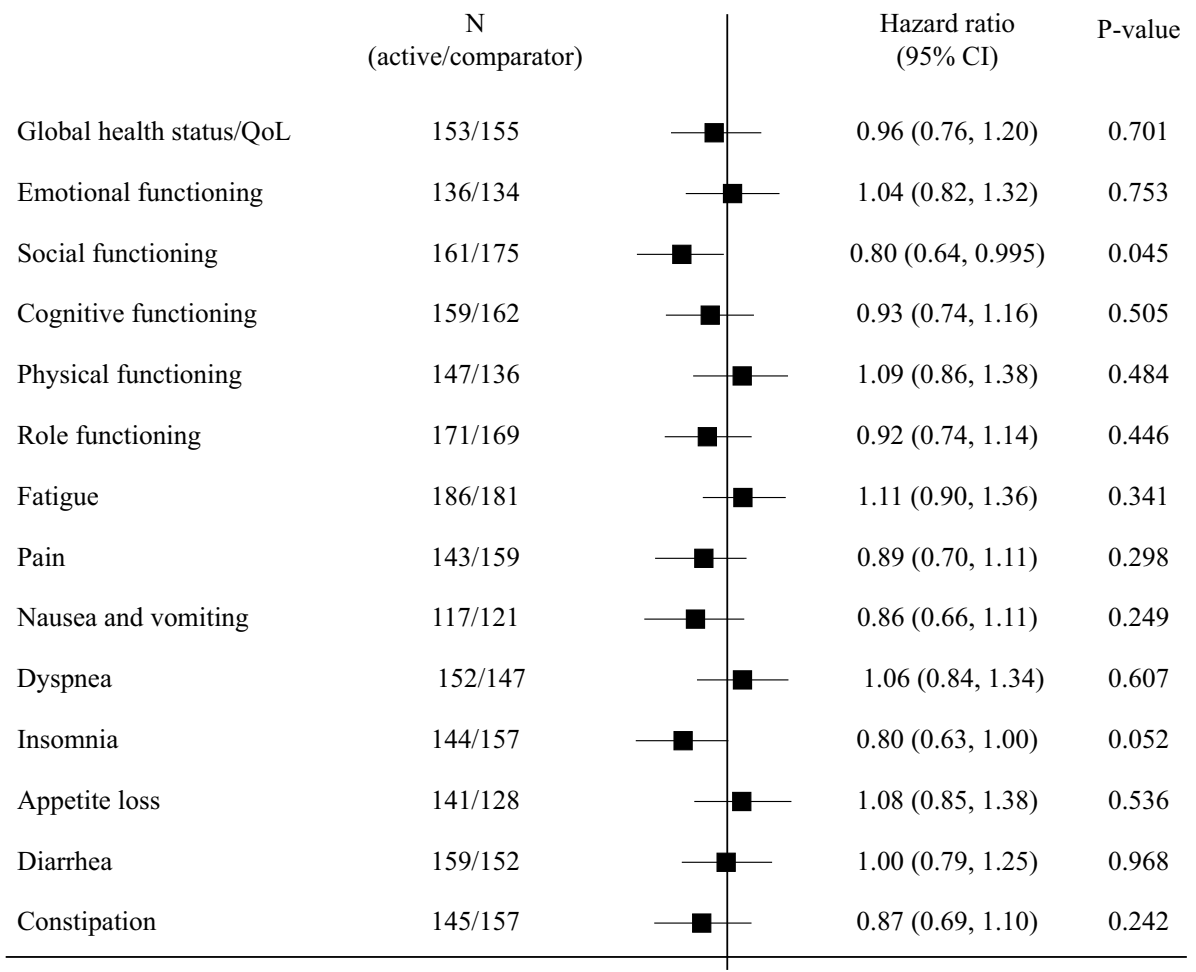

Favors daratumumab-based therapy $\longleftarrow 1.0 \longrightarrow$ Favors comparator

Fig. 3 Forest plot showing hazard ratios for differences between bortezomib/dexamethasone and carfilzomib-based therapy (ENDEAVOR trial) for time to $\geq 10$-point deterioration in subscales of the EORTC QLQ-C30. CI confidence interval, EORTC European Organisation for Research and Treatment of Cancer, $Q L Q-C 30$ EORTC 30-item Quality of Life Questionnaire, QoL quality of life

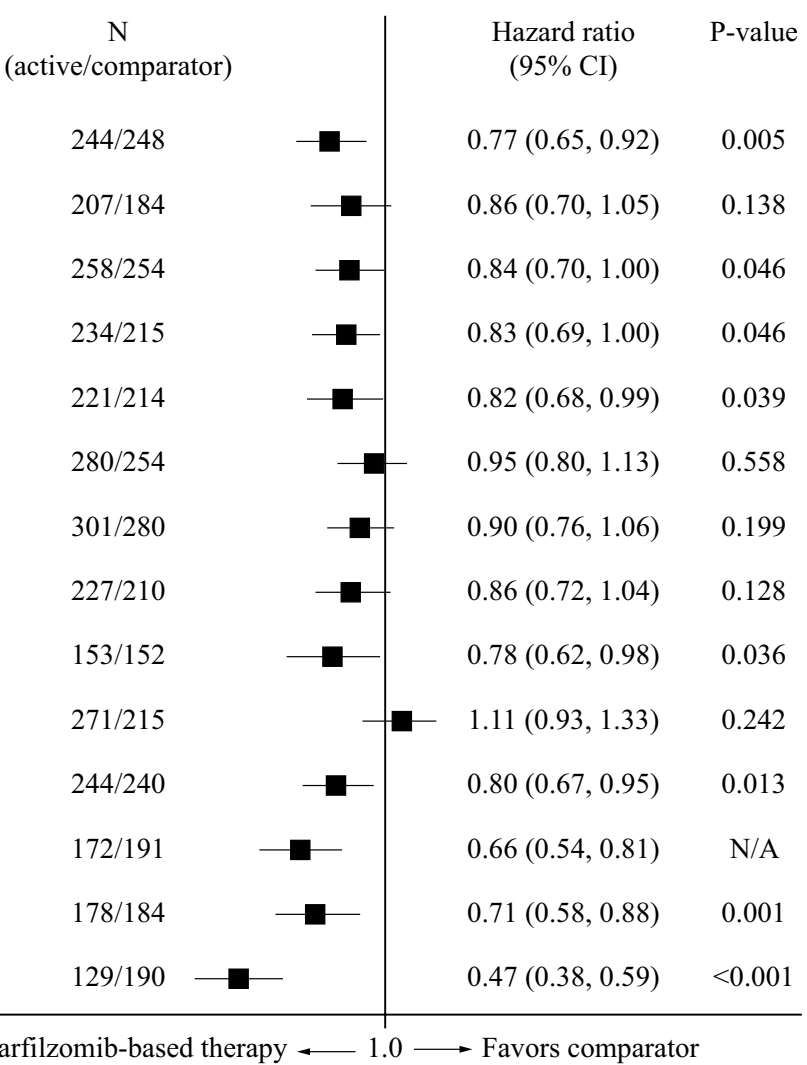

Favors carfilzomib-based therapy $\longleftarrow 1.0 \longrightarrow$ Favors comparator 
Fig. 4 Forest plot showing hazard ratios for differences between bortezomib/dexamethasone and daratumumab-based therapy (CASTOR trial) for time to $\geq 10$-point deterioration in subscales of the EORTC QLQ-C30. CI confidence interval, EORTC European Organisation for Research and EORTC 30-item Quality of Life Questionnaire, $Q o L$ quality of life Treatment of Cancer, $Q L Q-C 30$

\begin{tabular}{|c|c|c|c|c|}
\hline & $\begin{array}{c}\mathrm{N} \\
\text { (active/comparator) }\end{array}$ & & $\begin{array}{l}\text { Hazard ratio } \\
(95 \% \mathrm{CI})\end{array}$ & P-value \\
\hline Global health status/QoL & $123 / 122$ & $\square$ & $0.94(0.73,1.21)$ & 0.625 \\
\hline Emotional functioning & $113 / 113$ & & $0.82(0.63,1.08)$ & 0.151 \\
\hline Social functioning & $152 / 129$ & 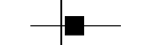 & $1.11(0.87,1.41)$ & 0.390 \\
\hline Cognitive functioning & $142 / 125$ & 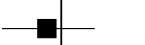 & $0.95(0.74,1.22)$ & 0.690 \\
\hline Physical functioning & $129 / 118$ & & $0.93(0.72,1.20)$ & 0.576 \\
\hline Role functioning & $152 / 133$ & 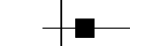 & $1.17(0.93,1.49)$ & 0.188 \\
\hline Fatigue & $166 / 146$ & . & $1.11(0.88,1.39)$ & 0.389 \\
\hline Pain & $141 / 121$ & & $1.01(0.79,1.29)$ & 0.954 \\
\hline Nausea and vomiting & $99 / 74$ & 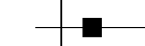 & $1.22(0.90,1.66)$ & 0.195 \\
\hline Dyspnea & $131 / 125$ & 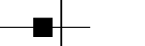 & $0.93(0.73,1.19)$ & 0.571 \\
\hline Insomnia & $134 / 117$ & 1 & $1.05(0.81,1.34)$ & 0.731 \\
\hline Appetite loss & $119 / 97$ & 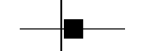 & $1.10(0.83,1.44)$ & 0.510 \\
\hline Diarrhea & $113 / 90$ & $a$ & $1.12(0.84,1.49)$ & 0.436 \\
\hline Constipation & $99 / 93$ & 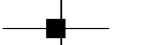 & $1.00(0.75,1.33)$ & 0.986 \\
\hline
\end{tabular}

$p<0.0001$ ) and 7.1 months (HR 0.33; 95\% CI 0.26, 0.43; $p<0.0001)$, respectively.

No major differences in safety outcomes were observed between study treatment and comparator in any of the four trials (Supplemental Table 2) with the exception of ENDEAVOR, in which the risk ratio $(95 \% \mathrm{CI})$ for time to Grade $\geq 2$ peripheral neuropathy was $0.11(0.07,0.16)$ in favor of carfilzomib/dexamethasone versus bortezomib/ dexamethasone $(p<0.0001)$. For Grade $\geq 3$ peripheral neuropathy, the risk ratio $(95 \% \mathrm{CI})$ was $0.16(0.08,0.31)$ $(p<0.0001)$.

\section{Discussion}

Patients with MM often experience impaired HRQoL [11-13]. For example, patients enrolled in the ASPIRE and ENDEAVOR studies had EORTC QLQ-C30 global health status/QoL scores of $~ 50-60$ of a maximum of 100 [24, $35,36]$. It is therefore important to avoid further deterioration wherever possible. In this analysis, HRQoL deteriorations were observed across all four phase III RRMM trials included here, although indirect, descriptive comparison across these trials suggests that the risk of HRQoL deterioration is less with carfilzomib-based than with daratumumabbased therapy. Results from the phase III ASPIRE and ENDEAVOR trials also show that a carfilzomib-based regimen has benefits over comparator treatments in many QoL domains of the EORTC QLQ-C30, including both functional and symptoms subscales. The benefit was particularly apparent on the global health status scale, where significant reductions of $23 \%$ and $21 \%$ in the risk of HRQoL deterioration were observed in ASPIRE and ENDEAVOR, respectively. In comparison, in POLLUX and CASTOR, the reductions in risk of global health status/QoL deterioration were $6 \%$ with daratumumab/lenalidomide/dexamethasone versus lenalidomide/dexamethasone and $4 \%$ with daratumumab/bortezomib/dexamethasone versus bortezomib/dexamethasone, respectively. In terms of the EORTC QLQ-C30 functional subscales, HRQoL benefits were observed for daratumumabbased treatment versus comparator only on social functioning. With respect to the individual symptoms scales, significant benefits in favor of carfilzomib-based therapy over comparators were seen for the constipation subscale in both the ASPIRE and ENDEAVOR trials, and additionally for the nausea/vomiting, insomnia, appetite loss, and diarrhea subscales in the ENDEAVOR trial. In contrast, there were no significant differences on the symptom subscales between daratumumab-based therapy and comparators in the POLLUX and CASTOR trials.

To put the results of this analysis in context with other published HRQoL trials in patients with RRMM, the TOURMALINE-MM1 study of lenalidomide/dexamethasone with or without ixazomib showed no differences in EORTC QLQ-C30 scores after a median of 23 months of follow-up, and no significant difference in OS was observed between 
treatments [17]. In the MM-003 study of pomalidomide plus low-dose dexamethasone versus high-dose dexamethasone, however, combination therapy was associated with a greater probability of improved HRQoL and prolonged time to HRQoL worsening [19]. The OS benefit in the subgroup of patients with $\geq 2$ prior treatments was 5 months. In the PANORAMA-1 study, HRQoL scores after 48 weeks of treatment with bortezomib/dexamethasone, with or without panobinostat, showed no benefit, and no significant difference in OS was observed between treatments [43].

Interestingly, it has been reported that there may be a relationship between improvement in HRQoL and treatment response in patients with cancer $[36,44]$. In a sub-analysis of the ENDEAVOR trial, patients receiving carfilzomib/ dexamethasone who were classified as responders (i.e., those with a partial response or better) experienced significant improvement on the EORTC QLQ-C30 global health status scale relative to non-responders [45].

All four trials included in the current analysis showed improved median PFS (the primary endpoint in each trial) for carfilzomib- or daratumumab-based therapy versus comparator [23, 24, 28, 29]. Furthermore, carfilzomib was associated with a median prolongation in OS of almost 8 months, regardless of whether it was used in combination with lenalidomide/dexamethasone or dexamethasone alone $[5,8,23$, 24, 28, 29]. Median PFS and OS have not yet been reached for daratumumab in published reports of final analyses, so it is unclear what magnitude of efficacy benefit is associated with this agent.

Tolerability was generally similar across the four trials $[23,24,28,29]$, although there was a significantly lower incidence of peripheral neuropathy symptoms of Grade $\geq 2$ with carfilzomib/dexamethasone (6\%) versus bortezomib/ dexamethasone $(32 \% ; p<0.0001)$ in the ENDEAVOR headto-head trial [23]. Indeed, bortezomib-induced peripheral neuropathy has a substantial impact on HRQoL and is difficult to manage, usually requiring reduction, interruption, or cessation of therapy [46].

To the best of our knowledge, this is the first descriptive comparison of HRQoL during carfilzomib- and daratumumab-based therapy, and the first time HRQoL has been assessed prospectively in a large cohort of similar patients with RRMM (carfilzomib $n>1700$; daratumumab $n>1000$ ). Another strength of the trials was the very high return rates for QoL questionnaires in a population of severely ill patients, for whom completion of QoL questionnaires may be considered too demanding and/or time consuming. In addition, some patients may have died or relapsed before questionnaires could be returned. There were, however, some limitations to the study. For example, this was an indirect descriptive comparison of carfilzomib- and daratumumab-based therapy, and direct, prospective, head-tohead trials are required to explore any potential differences between these agents in their impact on HRQoL and outcome. Furthermore, as the protocol-defined treatment cycles in which HRQoL was assessed differed between trials (see Table 1), this may have had an impact on the recorded time to deterioration. Any bias introduced in this way is expected to be minor, however, and mitigated by the use of HRs in these analyses. It is possible, however, that more frequent study visits could result in more intensive caregiver-patient interactions, with a positive impact on HRQoL. As these were prospective clinical trials, HRQoL measurements were made only during study treatment and stopped after disease progression or death. Another limitation was the potential for under-reporting of HRQoL impact as a result of different treatment durations between studies. For example, there was a maximum of eight cycles of daratumumab/bortezomib/ dexamethasone in the CASTOR study, after which patients received daratumumab monotherapy. There were also other methodological differences between trials, such as different patient numbers; dates of recruitment; patient characteristics; HRQoL instruments and cut-off points used, including protocol-defined MIDs; and pre- and post-study treatments. Moreover, the open-label designs of the four studies meant that patients were aware of their treatment assignment and response, which may have affected their answers to HRQoL questions, although no analysis of potential correlations between tumor responses and HRQoL were conducted as part of the AMNOG assessment.

In conclusion, this analysis has demonstrated the feasibility of HRQoL measurement with high questionnaire return rates in large populations of severely ill patients with RRMM. Currently, carfilzomib-based therapy is the only treatment for RRMM that has demonstrated significant differences versus study comparators in OS, global health status/QoL and other functional and symptom scales. Furthermore, descriptive analysis of the available HRQoL data across trials suggests potential benefits for carfilzomib-based therapy over standard therapy, as well as over daratumumabbased therapy. With the introduction of more effective therapies, the improvements in OS over time in patients with MM mean that the disease is now a chronic disorder, and so patients' HRQoL should be assessed regularly in prospective MM trials [47]. There may also be a role for QoL monitoring tools and apps, such as CANKADO (https://cankado.com/) to improve the quality of patient care.

Acknowledgements Editorial support was provided by Carine Thual of Amgen (Europe) $\mathrm{GmbH}$.

Author contributions All authors were involved in data analysis, writing, and approval of the paper.

Funding Medical writing assistance was provided by Dan Booth $\mathrm{PhD}$ (Bioscript Medical Ltd, Macclesfield, UK) and funded by Amgen (Europe) GmbH. The ASPIRE and ENDEAVOR studies discussed in 
this manuscript were originally sponsored by Onyx Pharmaceuticals, a subsidiary of Amgen Inc.

\section{Compliance with ethical standards}

Conflict of interest Katja Weisel has received honoraria from Amgen, BMS, Celgene, Janssen, Novartis, and Takeda; research funding from Amgen, Celgene, Janssen, and Sanofi; and has acted as a consultant for Amgen, Adaptive Biotech, BMS, Celgene, Janssen, Juno, Sanofi, and Takeda. Heinz Ludwig has received research funding from Amgen and Takeda; speaker bureau honoraria from Amgen, Takeda, JanssenCilag, BMS, and Celgene; and advisory board honoraria from Abbvie, Takeda, and Amgen. Achim Rieth and Andrea Lebioda are employees of Amgen GmbH and hold shares and stock options. Hartmut Goldschmidt has received research support from Amgen, BMS, Celgene, Chugai, Janssen, Sanofi, Mundipharma, Takeda, and Novartis; speaker bureau honoraria from ArtTempi, BMS, Celgene, Chugai, Janssen, and Novartis; and is a member of advisory boards for Adaptive Biotechnology, Amgen, BMS, Celgene, Janssen, Sanofi, and Takeda.

Open Access This article is distributed under the terms of the Creative Commons Attribution 4.0 International License (http://creativeco mmons.org/licenses/by/4.0/), which permits unrestricted use, distribution, and reproduction in any medium, provided you give appropriate credit to the original author(s) and the source, provide a link to the Creative Commons license, and indicate if changes were made.

\section{References}

1. Dingli, D., Ailawadhi, S., Bergsagel, P. L., Buadi, F. K., Dispenzieri, A., Fonseca, R., et al. (2017). Therapy for relapsed multiple myeloma: Guidelines from the Mayo Stratification for Myeloma and Risk-Adapted Therapy. Mayo Clinic Proceedings, 92(4), 578-598.

2. National Cancer Institute. (2019). Surveillance, Epidemiology, and End Results Program. Cancer Stat facts: Myeloma. Retrieved May 21, 2019, from https://seer.cancer.gov/statfacts/html/mulmy .html.

3. Wörmann, B., Driessen, C., Einsele, H., Goldschmidt, H., Gunsilius, E., \& Kortüm, M., et al. (2018). Multiple myeloma. Retrieved May 21, 2019, from https://www.onkopedia.com/de/onkopedia/ guidelines/multiples-myelom/@ @ view/html/index.html.

4. Fonseca, R., Abouzaid, S., Bonafede, M., Cai, Q., Parikh, K., Cosler, L., et al. (2017). Trends in overall survival and costs of multiple myeloma, 2000-2014. Leukemia, 31(9), 1915-1921.

5. Dimopoulos, M. A., Goldschmidt, H., Niesvizky, R., Joshua, D., Chng, W. J., Oriol, A., et al. (2017). Carfilzomib or bortezomib in relapsed or refractory multiple myeloma (ENDEAVOR): An interim overall survival analysis of an open-label, randomised, phase 3 trial. The lancet Oncology, 18(10), 1327-1337.

6. Facon, T., Dimopoulos, M. A., Dispenzieri, A., Catalano, J. V., Belch, A., Cavo, M., et al. (2018). Final analysis of survival outcomes in the phase 3 FIRST trial of up-front treatment for multiple myeloma. Blood, 131(3), 301-310.

7. Landgren, O., \& Iskander, K. (2017). Modern multiple myeloma therapy: Deep, sustained treatment response and good clinical outcomes. Journal of Internal Medicine, 281(4), 365-382.

8. Siegel, D. S., Dimopoulos, M. A., Ludwig, H., Facon, T., Goldschmidt, H., Jakubowiak, A., et al. (2018). Improvement in overall survival with carfilzomib, lenalidomide, and dexamethasone in patients with relapsed or refractory multiple myeloma. Journal of Clinical Oncology, 36(8), 728-734.

9. World Health Organization. (2018). WHOQOL: Measuring quality of life. Retrieved May 21, 2019, from https://www.who.int/ healthinfo/survey/whoqol-qualityoflife/en/.

10. International Society for Quality of Life Research. (2018). What is health-related quality of life research? http://www.isoqol.org/ about-isoqol/what-is-health-related-quality-of-life-research. Accessed May 21, 2019.

11. Gulbrandsen, N., Hjermstad, M. J., \& Wisloff, F. (2004). Interpretation of quality of life scores in multiple myeloma by comparison with a reference population and assessment of the clinical importance of score differences. European Journal of Haematology, $72(3), 172-180$.

12. Johnsen, A. T., Tholstrup, D., Petersen, M. A., Pedersen, L., \& Groenvold, M. (2009). Health related quality of life in a nationally representative sample of haematological patients. European Journal of Haematology, 83(2), 139-148.

13. Ramsenthaler, C., Osborne, T. R., Gao, W., Siegert, R. J., Edmonds, P. M., Schey, S. A., et al. (2016). The impact of diseaserelated symptoms and palliative care concerns on health-related quality of life in multiple myeloma: a multi-centre study. $B M C$ Cancer, 16, 427.

14. Sonneveld, P., Verelst, S. G., Lewis, P., Gray-Schopfer, V., Hutchings, A., Nixon, A., et al. (2013). Review of health-related quality of life data in multiple myeloma patients treated with novel agents. Leukemia, 27(10), 1959-1969.

15. Delforge, M., Dhawan, R., Robinson, D., Jr., Meunier, J., Regnault, A., Esseltine, D. L., et al. (2012). Health-related quality of life in elderly, newly diagnosed multiple myeloma patients treated with VMP vs. MP: Results from the VISTA trial. European Journal of Haematology, 89(1), 16-27.

16. Delforge, M., Minuk, L., Eisenmann, J. C., Arnulf, B., Canepa, L., Fragasso, A., et al. (2015). Health-related quality-of-life in patients with newly diagnosed multiple myeloma in the FIRST trial: Lenalidomide plus low-dose dexamethasone versus melphalan, prednisone, thalidomide. Haematologica, 100(6), 826-833.

17. Leleu, X., Masszi, T., Bahlis, N. J., Viterbo, L., Baker, B., Gimsing, P., et al. (2018). Patient-reported health-related quality of life from the phase III TOURMALINE-MM1 study of ixazomiblenalidomide-dexamethasone versus placebo-lenalidomide-dexamethasone in relapsed/refractory multiple myeloma. American Journal of Hematology, 93(8), 985-993.

18. Song, K. W., Dimopoulos, M. A., Weisel, K. C., Moreau, P., Palumbo, A., Belch, A., et al. (2015). Health-related quality of life from the MM-003 trial of pomalidomide plus low-dose dexamethasone versus high-dose dexamethasone in relapsed and/or refractory multiple myeloma. Haematologica, 100(2), e63-e67.

19. Weisel, K., Dimopoulos, M., Song, K. W., Moreau, P., Palumbo, A., Belch, A., et al. (2015). Pomalidomide and low-dose dexamethasone improves health-related quality of life and prolongs time to worsening in relapsed/refractory patients with multiple myeloma enrolled in the MM-003 randomized phase III trial. Clinical Lymphoma, Myeloma \& Leukemia, 15(9), 519-530.

20. Maes, H., \& Delforge, M. (2015). Optimizing quality of life in multiple myeloma patients: Current options, challenges and recommendations. Expert Review of Hematology, 8(3), 355-366.

21. Carlson, J. J., Guzauskas, G. F., Chapman, R. H., Synnott, P. G., Liu, S., Russo, E. T., et al. (2018). Cost-effectiveness of drugs to treat relapsed/refractory multiple myeloma in the United States. Journal of Managed Care \& Specialty Pharmacy, 24(1), 29-38.

22. Sparano, F., Cavo, M., Niscola, P., Caravita, T., \& Efficace, F. (2018). Patient-reported outcomes in relapsed/refractory multiple myeloma: A systematic review. Supportive Care in Cancer, 26(7), 2075-2090. 
23. Dimopoulos, M. A., Moreau, P., Palumbo, A., Joshua, D., Pour, L., Hajek, R., et al. (2016). Carfilzomib and dexamethasone versus bortezomib and dexamethasone for patients with relapsed or refractory multiple myeloma (ENDEAVOR): A randomised, phase 3, open-label, multicentre study. The lancet Oncology, 17(1), 27-38.

24. Stewart, A. K., Rajkumar, S. V., Dimopoulos, M. A., Masszi, T., Spicka, I., Oriol, A., et al. (2015). Carfilzomib, lenalidomide, and dexamethasone for relapsed multiple myeloma. New England Journal of Medicine, 372(2), 142-152.

25. Amgen Europe B.V. (2019). Kyprolis. Summary of product characteristics. Retrieved May 21, 2019, from https://www.medicines. org.uk/emc/product/5061.

26. Amgen Inc. (2019). Kyprolis. Prescribing information. Retrieved May 21, 2019, from https://www.pi.amgen.com/ /media/amgen/ repositorysites/pi-amgen-com/kyprolis/kyprolis_pi.pdf.

27. de Weers, M., Tai, Y. T., van der Veer, M. S., Bakker, J. M., Vink, T., Jacobs, D. C., et al. (2011). Daratumumab, a novel therapeutic human CD38 monoclonal antibody, induces killing of multiple myeloma and other hematological tumors. Journal of Immunology, 186(3), 1840-1848.

28. Dimopoulos, M. A., Oriol, A., Nahi, H., San-Miguel, J., Bahlis, N. J., Usmani, S. Z., et al. (2016). Daratumumab, lenalidomide, and dexamethasone for multiple myeloma. New England Journal of Medicine, 375(14), 1319-1331.

29. Palumbo, A., Chanan-Khan, A., Weisel, K., Nooka, A. K., Masszi, T., Beksac, M., et al. (2016). Daratumumab, bortezomib, and dexamethasone for multiple myeloma. New England Journal of Medicine, 375(8), 754-766.

30. Janssen-Cilag International NV. (2019). Darzalex. Summary of product characteristics. Retrieved May 21, 2019, from https:// www.medicines.org.uk/emc/product/7250.

31. Janssen Pharmaceutical Companies. (2019). Darzalex. Prescribing information. Retrieved May 21, 2019, from http://www.janssenlab els.com/package-insert/product-monograph/prescribing-infor mation/DARZALEX-pi.pdf.

32. Gemeinsamer Bundesausschuss. (2018). Beschluss des Gemeinsamen Bundesausschusses über eine Änderung der ArzneimittelRichtlinie (AM-RL): Anlage XII - Beschlüsse über die Nutzenbewertung von Arzneimitteln mit neuen Wirkstoffen nach § 35a SGB V - Daratumumab (neues Anwendungsgebiet; Neubewertung eines Orphan Drugs nach Überschreitung der 50 Mio. Euro Grenze). Retrieved May 21, 2019, from https://www.g-ba.de/ downloads/39-261-3222/2018-02-15_AM-RL_XII_Daratumuma b_D-310_BAnz.pdf.

33. Gemeinsamer Bundesausschuss. (2018). Beschluss des Gemeinsamen Bundesausschusses über eine Änderung der ArzneimittelRichtlinie (AM-RL): Anlage XII - Beschlüsse über die Nutzenbewertung von Arzneimitteln mit neuen Wirkstoffen nach $\S 35 \mathrm{a}$ SGB V - Carfilzomib (Neubewertung eines Orphan-Drugs nach Überschreitung der 50 Mio. Euro-Grenze). Retrieved May 21, 2019, from https://www.g-ba.de/downloads/39-261-3220/201802-15_AM-RL-XII_Carfilzomib_D-302_BAnz.pdf.

34. Dimopoulos, M. A., Moreau, P., Nahi, H., Plesner, T., Goldschmidt, H., Suzuki, K., et al. (2017). Efficacy and safety of daratumumab, lenalidomide, and dexamethasone versus $\mathrm{Rd}$ alone in relapsed or refractory multiple myeloma: Updated analysis of pollux. Haematologica, 102, 108-109.

35. Ludwig, H., Moreau, P., Dimopoulos, M. A., Mateos, M. V., Kaiser, M. F., Feng, S., et al. (2016). Health related quality of life results from the open-label, randomized, phase III ENDEAVOR trial evaluating carfilzomib and dexamethasone versus bortezomib and dexamethasone in patients with relapsed or refractory multiple myeloma. Blood, 128(Suppl 22), 3309.
36. Stewart, A. K., Dimopoulos, M. A., Masszi, T., Spicka, I., Oriol, A., Hajek, R., et al. (2016). Health-related quality-of-life results from the open-label, randomized, phase III ASPIRE trial evaluating carfilzomib, lenalidomide, and dexamethasone versus lenalidomide and dexamethasone in patients with relapsed multiple myeloma. Journal of Clinical Oncology, 34(32), 3921-3930.

37. Weisel, K., Lentzsch, S., Mateos, M. V., Hungria, V., Munder, M., Nooka, A., et al. (2017). Efficacy and safety of daratumumab, bortezomib and dexamethasone (DVD) versus bortezomib and dexamethasone (VD) in relapsed or refractory multiple myeloma (RRMM): Updated analysis of CASTOR. Haematologica, 102, 168-169.

38. Shih, C. L., Chen, C. H., Sheu, C. F., Lang, H. C., \& Hsieh, C. L. (2013). Validating and improving the reliability of the EORTC QLQ-C30 using a multidimensional Rasch model. Value in Health, 16(5), 848-854.

39. Kvam, A. K., Fayers, P. M., \& Wisloff, F. (2011). Responsiveness and minimal important score differences in quality-of-life questionnaires: A comparison of the EORTC QLQ-C30 cancerspecific questionnaire to the generic utility questionnaires EQ-5D and 15D in patients with multiple myeloma. European Journal of Haematology, 87(4), 330-337.

40. Snyder, C. F., Blackford, A. L., Sussman, J., Bainbridge, D., Howell, D., Seow, H. Y., et al. (2015). Identifying changes in scores on the EORTC-QLQ-C30 representing a change in patients' supportive care needs. Quality of Life Research, 24(5), 1207-1216.

41. Cocks, K., King, M. T., Velikova, G., Martyn St-James, M., Fayers, P. M., \& Brown, J. M. (2011). Evidence-based guidelines for determination of sample size and interpretation of the European Organisation for the Research and Treatment of Cancer Quality of Life Questionnaire Core 30. Journal of Clinical Oncology, 29(1), 89-96.

42. Osoba, D., Rodrigues, G., Myles, J., Zee, B., \& Pater, J. (1998). Interpreting the significance of changes in health-related qualityof-life scores. Journal of Clinical Oncology, 16(1), 139-144.

43. Richardson, P. G., Schlossman, R. L., Roy, A. N., Panneerselvam, A., Acharyya, S., Sopala, M., et al. (2018). Patient-reported outcomes of multiple myeloma patients treated with panobinostat after $\geq 2$ lines of therapy based on the international phase 3, randomized, double-blind, placebo-controlled PANORAMA-1 trial. British Journal of Haematology, 181(5), 628-636.

44. Dubois, D., Dhawan, R., van de Velde, H., Esseltine, D., Gupta, S., Viala, M., et al. (2006). Descriptive and prognostic value of patient-reported outcomes: The bortezomib experience in relapsed and refractory multiple myeloma. Journal of Clinical Oncology, 24(6), 976-982.

45. Siegel, D., Ludwig, H., Moreau, P., Mateos, M.-V., Niesvizky, R., \& Chng, W. J., et al. (2018). Association of clinical response with survival and quality of life after carfilzomib and dexamethasone (Kd56) treatment in the phase III ENDEAVOR trial. Presented at the European Hematology Association Annual Meeting, Stockholm, Sweden.

46. Berkowitz, A., \& Walker, S. (2012). Bortezomib-induced peripheral neuropathy in patients with multiple myeloma. Clinical Journal of Oncology Nursing, 16(1), 86-89.

47. Kvam, A. K., \& Waage, A. (2015). Health-related quality of life in patients with multiple myeloma-does it matter? Haematologica, 100(6), 704-705.

Publisher's Note Springer Nature remains neutral with regard to jurisdictional claims in published maps and institutional affiliations. 